\title{
From Matrices to Strings and Back
}

\author{
Shlomo S. Razamat* \\ C.N. Yang Institute for Theoretical Physics \\ State University of New York \\ Stony Brook, NY 11794-3840, U.S.A.
}

\begin{abstract}
We discuss an explicit construction of a string dual for the Gaussian matrix model. Starting from the matrix model and employing Strebel differentials techniques we deduce hints about the structure of the dual string. Next, following these hints a worldheet theory is constructed. The correlators in this string theory are assumed to localize on a finite set of points in the moduli space of Riemann surfaces. To each such point one associates a Feynman diagram contributing to the correlator in the dual matrix model, and thus recasts the worldsheet expression as a sum over Feynman diagrams.
\end{abstract}

KeYwords: AdS/CFT, Matrix models.

\footnotetext{
*Email: razamat@max2.physics.sunysb.edu
} 


\section{Contents}

1. Introduction 1

2. From matrices to strings 1

2.1 The matrix model 1

2.2 Extracting hints about the dual theory

3. The string theory 7

3.1 Constructing the action 0

3.2 Integration over the metric moduli 11

3.3 Operators in the string theory 11

3.4 Definition of $g_{D}$ and $\Delta$ at the Strebel points 12

4. From strings to matrices 15

5. Summary 19

A. A short primer on Strebel differentials 20

B. A localization mechanism 22

\section{Introduction}

One of the most fruitful directions of research in the recent years has been the exploration of the connections between gravity theories in higher dimensions, string theory in particular, and gauge theories.

Although a significant number of gauge/string dualities is well established by now, we still are lacking a "microscopic" understanding of the mechanisms behind the dualities in most cases. In cases when such mechanism is well understood we do not know how to apply it to more general setups. Examples of these mechanisms are the ChernSimons/topological string duality [1, 2], the Kontsevich model [3, 4, 5, 6], and the double scaled matrix models $/ c \leq 1$ string theory duality [7, 8]. See also [9, 10, 11, 12] for a recent discussion of the $A d S_{5} / C F T_{4}$ duality.

The "language" naturally spoken by a perturbative gauge theory is that of sums of Feynman graphs. On the other hand the "language" spoken by a string theory is that of integrals over moduli space of punctured Riemann surfaces. The problem of having 
a microscopic understanding of a given instance of gauge/string duality ultimately boils down to finding a dictionary between the two languages. That is, either figuring out how Feynman diagrams arise from a worldsheet description of a string theory, or alternatively constructing a worldsheet from Feynman diagrams. For instance, in the "topological" duality of [1, [] the diagrams appear on the string side as different classical solutions of the worldsheet theory. In case of double scaled matrix model [7], 8] one obtains a string worldsheet by taking the large $N$ limit and tuning the couplings of the theory so that large Feynman diagrams dominate and effectively can be treated as a discretization of a string worldsheet.

There exists a mathematical tool which can facilitate the translation between Feynman graphs and moduli spaces of Riemann surfaces. Using a special class of quadratic differentials on a Riemann surface, the Strebel differentials, one can establish an isomorphism between the space of metric graphs with a given number of faces and the moduli space of punctured Riemann surfaces [13, 14, 15]. This tool has been used in different contexts in the framework of string theory, such as string field theory (see for example [16, 17]) and matrix models (see [18] for a review). Strebel differentials played an important role in understanding the Kontsevich model $/ 2 d$ topological gravity duality $\llbracket$, although by now we have additional ways to obtain microscopic understanding of this duality [19, 20].

In [21] it was suggested to use Strebel differentials to explicitly construct a generic class of gauge/string dualities in the free limit of the gauge theory side of the correspondence. See [22, 23] for earlier work and [24, 25, 26, 27, 28, 29] for subsequent developments. The logic of this prescription is as follows. The isomorphism between moduli spaces and graphs implied by Strebel differentials needs a metric to be defined for the graphs. The choice of metric effects the properties of the worldsheet dual. In [21] R. Gopakumar introduced a metric on the Feynman graphs through Schwinger parameters. One can write Feynman diagrams in a given field theory as integrals over Schwinger parameters. Then, using Strebel differentials this integration can be transformed to an integration over moduli space of punctured Riemann surfaces. The integrand of this integral is interpreted as a worldsheet correlator of a $2 d C F T$ dual of the field theory. By computing enough worldsheet correlators in this way one hopes to gather enough evidence to deduce what is actually the string theory producing them.

Although the prescription of 21] is very explicit it is technically hard to implement in practice. Even in simplest cases making the transformation of measure between Schwinger and moduli parameters eventually reduces to solving elliptic integral equations which is usually impossible to do analytically. ${ }^{1}$ Thus, it is hard to gather substantial amount of information from the correlators to completely specify the string dual.

The main purpose of this paper is to discuss this program in a simplified setting. We make two simplifications. First, the field theory side is taken to be simply a (Gaussian)

\footnotetext{
${ }^{1}$ However, some correlators have been explicitly computed 25, 26, 27.
} 
matrix model. Second, we change the metric on the Feynman graphs.

There is a natural metric on the Feynman graph which does not require any additional structure to be introduced [30]. We can assign a unit of length to every edge of the diagram. Consider the following correlator in some gauge theory

$$
\left\langle\prod_{j=1}^{s} \operatorname{Tr} Q^{J_{j}}\right\rangle
$$

where $\operatorname{Tr} Q^{J}$ is a composite of $J$ adjoint fields in the theory. Then the set of all graphs dual to the connected diagrams contributing to the calculation in the free limit is the set of metric graphs with $s$ faces with circumferences of the faces being $J_{j}$. Each diagram will be accompanied by an appropriate numerical factor computed as the number of the different Wick contractions giving this graph and appropriate momenta (position) dependence. Taking the gauge theory to be a matrix model one gets rid of the momentum (position) dependencies. Using the Strebel theorem ${ }^{2}$ one can map each Feynman graph to a point in the moduli space of Riemann surfaces $\mathcal{M}_{\mathrm{g}, s}$. Thus, the correlator above can be seen as a weighed sum over discrete set of points in the moduli space. However, in a usual string theory the worldsheet correlators are given in terms of smooth integrals over the moduli space, and we will have to reconcile this issue.

In what follows we will construct a string dual of the Gaussian matrix model which will exhibit the properties discussed above. The worldsheet theory will consist of three parts: a $c=1$ and a $c=-23$ CFTs which will be explicitly specified, and a $c=48$ CFT which will only be implicitly defined through some of its properties . The correlators of the $c=48 \mathrm{CFT}$ will be assumed to localize the moduli space integration to a set of points corresponding to Strebel differentials with integer edge lengths. ${ }^{3}$ We will refer to these special locations as Strebel points. As we will discuss in the bulk of the paper the Strebel points are in one to one correspondence with the Feynman diagrams contributing to a given correlator. We will not be able to provide an explicit mechanism for such a localization. However, assuming this property the sphere string theory correlators will be shown to reproduce planar matrix model results.

The paper is organized as follows. In section 2 we review the results of [30] and in particular starting from Gaussian matrix model and using Strebel differential techniques gather some hints about its stringy dual. Following these hints in section 3 we construct a worldsheet model. In section 1 we show that certain correlators in this model reproduce the matrix model results. Finally, in section 5 we summarize our results. A short primer on Strebel differentials is included in appendix A. In appendix B we discuss a heuristic suggestion for the localization mechanism.

\footnotetext{
${ }^{2}$ We refer the reader to appendix $\mathrm{A}$ or to [21, 27, 30] for brief expositions of the Strebel theorem.

${ }^{3}$ For a similar discussion in the case of a symmetric product orbifolds see 31.
} 


\section{From matrices to strings}

In this section we discuss the Gaussian matrix model and the hints it provides about its stringy dual through Strebel differentials.

\subsection{The matrix model}

We consider a gauge invariant correlator (1.1) computed in the Gaussian matrix model with an action given by

$$
S_{M}=\frac{1}{2} t N \operatorname{Tr} Q^{2}
$$

where $Q$ is a hermitian $N \times N$ matrix.

This correlator is computed as a sum of the different Wick contractions. It can be naturally expanded in powers of $N$. Every loop gives a factor of $N$, and a propagator gives $(N t)^{-1}$. All the vertices are external and do not give any factors of $N$. Thus the power of $N$ accompanying each diagram contributing to an $s$ point correlator (1.1) is $f-e=2-2 \mathrm{~g}-s$

( $f$ is the number of loops and $e$ is the number of edges in a given diagram). Thus, a correlator (1.1) in the free theory can be written as

$$
t^{-\frac{1}{2} \sum_{j=1}^{s} J_{s}} \sum_{i} N^{2-2 \mathrm{~g}_{i}-s} C_{i}=\prod_{k=1}^{s} J_{k} \prod_{J=1}^{\infty} v_{J} ! N^{-s} t^{-\frac{1}{2} \sum_{j=1}^{s} J_{s}} \sum_{i} \frac{N^{2-2 \mathrm{~g}_{i}}}{\#\left(\Gamma_{i}\right)}
$$

where summation is over different Feynman graphs and $C_{i}$ is the number of Wick contractions giving each graph (for instance see [32]). $v_{J}$ is the number of vertices of power $J . \mathrm{g}_{i}$ is the genus and the factor $\#\left(\Gamma_{i}\right)$ is the symmetry factor of the $i$ th Feynman graph (we identify the graphs by mapping the set of vertices and the set of edges onto themselves keeping the orientation of the vertices fixed).

\subsection{Extracting hints about the dual theory}

In this section we briefly review the construction of [30]. The starting point is a variation of Gopakumar's prescription for constructing a string theory dual for a free field theory [21]. In this variation we seek a string theory with the following property. For a given correlator the integration over the moduli space of the Riemann surfaces localizes to a discrete set of points, with every point corresponding to a Feynman diagram contributing to the calculation on the field theory side. Given a Feynman diagram the corresponding point in the moduli space is computed through Strebel differentials by the following simple procedure. We associate a unit of length to each edge of the Feynman diagram. Next, we make a skeleton graph from the Feynman diagram [21] by gluing the homotopically equivalent edges together. The length of the edges of the skeleton graph is the number of edges of the Feynman graph glued together. Then, the point in the moduli space corresponding to the Feynman diagram is the unique point corresponding to the Strebel 
differential with the dual of the skeleton graph being its critical curve and the metric on the graph given by the lengths of the edges defined above.

The above construction by itself seems to teach us very little about the string theory side. However, one can extract some clues about the string theory through the following procedure. Let us devise a "caricature" of string theory which will capture the Strebel differential construction above and have some string theory structure to it. The correlators in the "caricature" model are computed using the following expression

$$
\left\langle\prod_{k=1}^{s} \mathcal{O}_{J_{k}}\right\rangle_{\mathrm{g}}=\left[\sum_{\varphi \in \mathcal{S}_{\mathrm{g}, s}} \frac{1}{\Gamma(\varphi)}\right]\left[\prod_{k \in Z(\varphi) \cup P(\varphi)} \int_{0}^{1} d X_{k}\right] e^{-S} \prod_{k=1}^{s} \mathcal{O}_{J_{k}} .
$$

The first square brackets is a toy model for integration over moduli space. The set $\mathcal{S}_{\mathrm{g}, s}$ is the set of all Strebel differentials, $\varphi$, on genus g surface with $s$ punctures with integer edges. ${ }^{4} \Gamma(\varphi)$ is a discrete measure on the moduli space to be specified shortly. The second bracket gives a toy integration over a worldsheet "field" $X . X$ is defined only at the poles, $P(\varphi)$, and zeros, $Z(\varphi)$, of the differential $\varphi$. The toy action, $S$, is given by

$$
S \equiv 2 \pi i \sum_{k \in P(\varphi) \cup Z(\varphi)}\left[p_{k}(\varphi)-2\right] X_{k}-N \sum_{k \in Z(\varphi)} e^{-4 \pi i X_{k}}
$$

with $p(\varphi)$ being the residues of the corresponding pole or zero of $\varphi$. For a double pole it is the usual residue of the square root of the differential and for a zero $p=0$. The operators are defined as $^{5}$

$$
\operatorname{Tr} Q^{J} \rightarrow \mathcal{O}_{J}=N^{-J / 2} J \sum_{k \in P(\varphi)} e^{2 \pi i(J-2) X_{k}}
$$

We specify $\Gamma(\varphi)$ to be

$$
\Gamma(\varphi) \equiv \# \Gamma(\varphi) \times n(\varphi)
$$

where $\# \Gamma(\varphi)$ is the symmetry factor of the critical curve of $\varphi$ and $n(\varphi)$ is the number of points on the moduli space having the same graph as their critical curve. ${ }^{6}$

With the above definitions it is easy to convince oneself that the correlator computed on the matrix theory side, $\left\langle\prod_{j=1}^{s} \operatorname{Tr} Q^{J_{j}}\right\rangle_{\mathrm{g}}$ is exactly equal to the correlator $\left\langle\prod_{k=1}^{s} \mathcal{O}_{J_{k}}\right\rangle_{\mathrm{g}}$ computed in the "caricature" string theory model. Strictly speaking the toy model gives the sum over connected Feynman diagrams lacking homotopically trivial self contractions. If so desired, the homotopically trivial self contractions can be easily taken into account by

\footnotetext{
${ }^{4}$ See e.g. 33, 34, 35 for discussion of Strebel differentials with integer edges.

${ }^{5}$ In this toy model we will not be able to incorporate the dilaton operator, $\operatorname{Tr} Q^{2}$.

${ }^{6}$ Note that there is a unique Strebel differential for every graph with labeled vertices. However, in the above construction we do not label the vertices and thus there are several points corresponding for a given graph.
} 
either redefining the set $\mathcal{S}_{\mathrm{g}, s}$ or by redefining the operators $\mathcal{O}_{J}$ [30]. The integration over the fields $X_{i}$ does not vanish only for differentials which have the set $J_{i}$ as the set of the residues of their double poles. Because we restrict ourselves to differentials with integer edges, all these differentials correspond to the Feynman diagrams contributing to (1.1). The integration can be easily performed and the equivalence established.

Next, one can recast the toy model above into a bit more familiar language. The first term in the action (2.4) can be written in the following form

$$
2 \pi i \sum_{k \in P(\varphi) \cup Z(\varphi)}\left[p_{k}(\varphi)-2\right] X_{k}=-i \int d^{2} z \sqrt{g_{D}} R_{D} X(z, \bar{z}),
$$

where the field $X(z, \bar{z})$ is equal to $X_{k}$ at the special points of the differential and the metric $g_{D}$ is defined as follows. Given a Strebel differential $\varphi$ with integer edges we can define a dual Strebel differential as (see section 3.4 and [30] for more details)

$$
\varphi_{D}=-\frac{\varphi}{\sin ^{2} \pi l}, \quad l(z)=\int_{z_{0}}^{z} \sqrt{\varphi} d z,
$$

where $z_{0}$ is some (does not matter which) zero of $\varphi$. The metric then is simply given by

$$
g_{D}=\left|\varphi_{D}\right| \text {. }
$$

Moreover, the second term in (2.4) is heuristically of the following form

$$
N \sum_{k \in Z(\varphi)} e^{-4 \pi i X_{k}} \rightarrow \mu \int d^{2} z \sqrt{g_{D}} e^{-4 \pi i X(z, \bar{z})} .
$$

Thus, the action can be schematically written as

$$
S \sim-i \int d^{2} z \sqrt{g_{D}} R_{D} X(z, \bar{z})-\mu \int d^{2} z \sqrt{g_{D}} e^{-4 \pi i X(z, \bar{z})} .
$$

Adding a kinetic term of time like boson to the above action we get a $c=25 C F T$. To see this we proceed as follows. For the toy model to reproduce the matrix model results the ratio between the coefficients in the exponent and the coefficient of the linear term has to be $4 \pi$, as it is in (2.11). In the background charge representation of conformal field theories with time like field,

$$
S=\frac{1}{4 \pi} \int d^{2} z\left[-\partial \phi \bar{\partial} \phi-\frac{1}{2} i Q \sqrt{g} R \phi+\mu \sqrt{g} e^{i b \phi}\right],
$$

these coefficients are related due to conformal invariance by

$$
Q=-\frac{2}{b}-b
$$

Here $Q$ is the background charge and it is related to the central charge through $c=1+3 Q^{2}$. We can rescale the field $X$ by some constant $a^{-1}$ to obtain the relation (2.13). We get the following equation

$$
8 \pi a=\frac{2}{4 \pi a}+4 \pi a \rightarrow|a|=\frac{1}{2 \sqrt{2} \pi}
$$


Thus we deduce that in our case $Q=2 \sqrt{2}$ and $c=25$. If one tries a space-like sign for the field $X$ then we again obtain $c=25$ but with the field $X$ rescaled by an imaginary number. As we have taken $X$ to be real in (2.11) we are forced to consider $X$ to be time-like.

The model above is a toy model without any obvious or direct relation to a string theory construction. However, assuming that there is a string theory with properties described in the beginning of this section one can try and extract some hints about this theory from the toy model above. In the following sections we will try to construct a string theory dual of the Gaussian matrix models starting from the following clues extracted from the toy model,

- The worldsheet theory contains a version of the $c=25$ CFT obtained in (2.11).

- The metric $g_{D}$ might come handy in trying to prove that the string theory discussed is related to a matrix model. By definition a string theory is independent on a particular choice of the worldsheet metric due to Weyl invariance so we will have to understand what role the special metric $g_{D}$ might play.

We would like to stress that we will not try to reproduce the toy model from a well defined string theory but rather follow the clues above to understand how to build a stringy dual for the Gaussian matrix model.

\section{The string theory}

We will now follow the hints of the previous section to construct a worldsheet dual for the Gaussian matrix model. The string theory consists of three pieces: $\tilde{X}$ CFT with central charge $c=-23, Y$ CFT with $c=1$, and the $\chi$ CFT with $c=48$. The logic is as follows. First, we will start with the worldsheet model motivated by the construction of the previous section. This model consists of a $c=25 \hat{X}$ CFT and a $c=1 \hat{Y}$ CFT. The $c=25 \hat{X}$ CFT is the one obtained from the toy model construction of the previous section, and the $c=1$ $\hat{Y}$ CFT is introduced to render the total central charge of the matter fields to be $c=26$. After performing some elementary field redefinitions we will make a non trivial change of sign of one of the kinetic terms to recast the worldsheet action as a sum of $c=-23$ $X \mathrm{CFT}, c=1 Y \mathrm{CFT}$, and a $c=48$ measure on the moduli space. We then interpret the $c=48$ measure as a result of computing a correlator of a $c=48 \chi \mathrm{CFT}$. Assuming that the moduli space integration localizes to the Strebel points, in the next section the worldsheet model obtained in this fashion is shown to reproduce planar Gaussian matrix model correlators.

\subsection{Constructing the action}

We start the construction from the following $c=26$ worldsheet theory. The matter content consists of two CFTs, which we will denote as $\hat{X}$ and $\hat{Y}$. The fields $\hat{X}$ and $\hat{Y}$ are taken to 
be periodic with period 1 . The action is defined as

$$
\begin{aligned}
& S_{\hat{X}}(\hat{\mu})=-2 \pi \int d^{2} z \partial \hat{X} \bar{\partial} \hat{X}-i \int d^{2} z \sqrt{g_{B}} R_{B} \hat{X} . \\
& S_{\hat{Y}}(\hat{\tau})=2 \pi \int d^{2} z \partial \hat{Y} \bar{\partial} \hat{Y} .
\end{aligned}
$$

The $\hat{X} C F T$ has central charge 25 and the $\hat{Y} C F T$ has unit central charge. ${ }^{7}$ The metric $g_{B}$ is the "dynamical" metric with respect to which the measure of the path integral is defined. In what follows we will assume that $g_{B}$ is in a conformal gauge and has the following form

$$
g_{B}(z, \bar{z}) d z d \bar{z}=\left|\varphi_{B}(z)\right| d z d \bar{z}
$$

where $\varphi_{B}$ is a meromorphic function on the worldsheet. Let us discuss this action in detail.

\section{The $X C F T$}

The $\hat{X} C F T$ has central charge $c=25$. One way to see this is by making a Weyl transformation, $g_{B} \rightarrow e^{2 \omega} g_{B}$. Under this transformation the field $\hat{X}$ transforms as

$$
\hat{X} \rightarrow \hat{X}-\frac{i}{\pi} \omega, \quad \sqrt{g_{B}} R_{B} \rightarrow \sqrt{g_{B}} R_{B}-4 \partial \bar{\partial} \omega
$$

Thus we get

$$
\delta S_{\hat{X}}=-\frac{2}{\pi} \int d^{2} z \partial \omega \bar{\partial} \omega-\frac{1}{\pi} \int d^{2} z \sqrt{g_{B}} R_{B} \omega=-24 S_{L}(\omega)
$$

where

$$
S_{L}(\omega)=\frac{1}{12 \pi} \int d^{2} z\left[\partial \omega \bar{\partial} \omega+\frac{1}{2} \sqrt{g_{B}} R_{B} \omega\right] .
$$

The measure of integration, $[\mathcal{D} \hat{X}]_{g_{B}}$ gives another factor of $S_{L}$ and thus we get that the $\hat{X}$ CFT has $c=25$.

Note that we defined $\hat{X}$ to be a periodic scalar of period one, and this is in odds with the Weyl transformation above. Thus, the field $\hat{X}$ should generally have some imaginary part. We make the following field redefinition

$$
\hat{X} \equiv X+\frac{i}{2 \pi} \ln \left|\frac{\varphi_{D}}{\varphi_{B}}\right| \equiv X+\frac{i}{\pi} \hat{\omega} .
$$

Here, $\left|\varphi_{D}\right|\left(\equiv g_{D}\right)$ is some moduli dependent metric which does not transform under Weyl transformations. This metric is related to the one discussed in the previous section and we will carefully define it below. The field $X$ is also $W e y l$ invariant and will be the one integrated over, i.e. $[\mathcal{D} \hat{X}]_{g_{B}} \rightarrow[\mathcal{D} X]_{g_{B}}$. Using this definition the $X$ field is periodic with

\footnotetext{
${ }^{7}$ This worldsheet matter content was discussed by Itzhaki and McGreevy [36] as a candidate for a dual of large $N$ gauged harmonic oscillator [37. One can transform to actions with usual kinetic terms by taking $\phi=-2 \sqrt{2} \pi \hat{X}$.
} 
period 1 and has $c=25$. One can think of the redefinition of $\hat{X}$ merely as specifying the metric for which $\hat{X}$ has no imaginary part. This redefinition will become non trivial shortly for two reasons. First, the operators will be defined through fields $X$ and not $\hat{X}$ and second we will turn the field $X$ into a space-like field. We observe the following,

$$
\begin{aligned}
S_{\hat{X}}(0) & =-2 \pi \int d^{2} z \partial\left(X+\frac{i}{\pi} \hat{\omega}\right) \bar{\partial}\left(X+\frac{i}{\pi} \hat{\omega}\right)-i \int d^{2} z \sqrt{g_{B}} R_{B}\left(X+\frac{i}{\pi} \hat{\omega}\right)= \\
& =-2 \pi \int d^{2} z \partial X \bar{\partial} X-i \int d^{2} z \sqrt{g_{D}} R_{D} X+\frac{2}{\pi}\left[\int d^{2} z \partial \hat{\omega} \bar{\partial} \hat{\omega}+\frac{1}{2} \int d^{2} z \sqrt{g_{B}} R_{B} \hat{\omega}\right] \\
& =-2 \pi \int d^{2} z \partial X \bar{\partial} X-i \int d^{2} z \sqrt{g_{D}} R_{D} X+24 S_{L}\left(\varphi_{B} \rightarrow \varphi_{D}\right)
\end{aligned}
$$

where $S_{L}\left(\varphi_{B} \rightarrow \varphi_{D}\right)=S_{L}(\hat{\omega})$ is the Liouville action for going from metric $g_{B}$ to $g_{D}$. Under Weyl transformations $g_{D}$ and $X$ are held fixed and $g_{B}$ transforming as usual. Thus, under Weyl transformation $g_{B} \rightarrow \exp \left(2 \omega^{\prime}\right) g_{B}$ the action transforms as

$$
\delta S_{\hat{X}}(0)=24 S_{L}\left(e^{2 \omega^{\prime}} \varphi_{B} \rightarrow \varphi_{D}\right)-24 S_{L}\left(\varphi_{B} \rightarrow \varphi_{D}\right)=-24 S_{L}\left(\varphi_{B} \rightarrow e^{2 \omega^{\prime}} \varphi_{B}\right) .
$$

Note that the field $X$ is time like and because it is Weyl invariant, we can change the sign of the kinetic term of $X$ (not of $\hat{X}$ ) without changing the central charge of the CFT. We will treat the field $X$ as spacelike and define

$$
\begin{aligned}
S_{L} & =S_{L}\left(\varphi_{B} \rightarrow \varphi_{D}\right), \\
S_{X}(\hat{\mu}) & =2 \pi \int d^{2} z \partial X \bar{\partial} X-i \int d^{2} z \sqrt{g_{D}} R_{D} X-\hat{\mu} \mathcal{O}_{0} .
\end{aligned}
$$

The operator $\mathcal{O}_{0}$ will be defined in section 3.3. Note that the theory $S_{X}+S_{L}$ is not equivalent to $S_{\hat{X}}$ because of the change of the sign of the kinetic term. We merely used $\hat{X}$ action to construct the action of our interest. It is most natural to think of the $X C F T$ as a $c=-23$ usual space-like theory with background charge accompanied by a measure factor with Weyl anomaly $c=48$. This comes about as follows. We define

$$
S_{X}^{(\hat{\omega})}=S_{X}(\hat{\mu}=0)+24 S_{L}(\hat{\omega}), \quad \hat{\omega}=\frac{1}{2} \ln \left|\frac{\varphi_{D}}{\varphi_{B}}\right|,
$$

and we can rewrite this as

$$
S_{X}^{(\hat{\omega})}=2 \pi \int d^{2} z \partial X \bar{\partial} X+4 i \int d^{2} z \partial \bar{\partial} \hat{\omega} X-i \int d^{2} z \sqrt{g_{B}} R_{B}\left(X+\frac{i}{\pi} \hat{\omega}\right)+\frac{2}{\pi} \int d^{2} z \partial \hat{\omega} \bar{\partial} \hat{\omega} .
$$

At this point $\hat{\omega}$ can be a generic scalar function on the worldsheet transforming under Weyl $g_{B} \rightarrow e^{2 \omega} g_{B}$ as $\hat{\omega} \rightarrow \hat{\omega}-\omega$. We can redefine the field $X$ by

$$
\tilde{X} \equiv X-\frac{i}{\pi} \hat{\omega}
$$


Using this definition the action becomes

$$
\begin{aligned}
S_{X}^{(\hat{\omega})} & =2 \pi \int d^{2} z \partial \tilde{X} \bar{\partial} \tilde{X}-i \int d^{2} z \sqrt{g_{B}} R_{B} \tilde{X}+\frac{4}{\pi} \int d^{2} z\left[\partial \hat{\omega} \bar{\partial} \hat{\omega}+\frac{1}{2} \sqrt{g_{B}} R_{B} \hat{\omega}\right] \\
& =S_{\tilde{X}}+48 S_{L}(\hat{\omega}) .
\end{aligned}
$$

Thus, we decompose the $c=25 X C F T$ into a $c=-23 C F T \tilde{X}$ and a measure which has Weyl anomaly $c=48$. Thus we can think of the $X$ action as $c=-23 C F T$ and a Weyl non invariant measure on the moduli space. The $X$ action (3.9) is equivalent to the $\tilde{X}$ action (3.13) and for computational reasons it will be more convenient to use the $X$ action in what follows.

\section{The $Y C F T$}

For the $Y C F T$ we make a trivial redefinition of the field,

$$
\hat{Y} \equiv Y-\frac{i}{2 \pi} \ln |\Delta|
$$

where $\Delta$ is some moduli dependent function on the worldsheet to be specified later. We obtain

$$
\begin{aligned}
S_{\hat{Y}}(0) & =2 \pi \int d^{2} z \partial\left(Y-\frac{i}{2 \pi} \ln |\Delta|\right) \bar{\partial}\left(Y-\frac{i}{2 \pi} \ln |\Delta|\right)= \\
& =2 \pi \int d^{2} z \partial Y \bar{\partial} Y+2 i \int d^{2} z(\partial \bar{\partial} \ln |\Delta|) Y-\frac{1}{2 \pi} \int d^{2} z \partial \ln |\Delta| \bar{\partial} \ln |\Delta| .
\end{aligned}
$$

This is a $c=1 C F T$ as the action is independent on the metric. We will define

$$
\begin{aligned}
S_{\Delta} & =-\frac{1}{2 \pi} \int d^{2} z \partial \ln |\Delta| \bar{\partial} \ln |\Delta| \\
S_{Y}(\hat{\tau}) & =2 \pi \int d^{2} z \partial Y \bar{\partial} Y+2 i \int d^{2} z(\partial \bar{\partial} \ln |\Delta|) Y-\hat{\tau} \mathcal{O}_{-2} .
\end{aligned}
$$

The operator $\mathcal{O}_{-2}$ will be defined in section 3.3. The above redefinition of the field $\hat{Y}$ will become non trivial later on, as the definition of vertex operators will depend on the fields $Y$. Thus, the explicit dependence on $\Delta$ contributes to the non standard measure on the moduli space.

To summarize, the matter action of our string theory is

$$
S=S_{X}(\hat{\mu})+S_{Y}(\hat{\tau})+24 S_{L}+S_{\Delta} .
$$

We regard this action as a combination of a $c=-23$ and $c=1$ CFTs together with a $c=48$ measure. We assume that the $c=48$ part (with the measure dependent factors of section 3.3) can be obtained as correlators in some $c=48$ CFT. 


\subsection{Integration over the metric moduli}

From now on we restrict our discussion to the planar case, i.e. the worldsheet is a sphere. To compute the following correlator,

$$
\left\langle\prod_{k=1}^{s} \mathcal{O}_{J_{k}}\right\rangle_{\mathrm{g}=0}
$$

we have to fix the $S L(2, \mathbb{C})$ symmetry on the sphere. To do so we fix the positions of three of the operators. The correlator has insertions with $s$ quantum numbers $J_{i}$. For each point of the moduli space, i.e. for each set of positions of the unfixed operators, there is a unique Strebel differential that has double poles at the positions of the operators with residues $J_{k}$. We denote this differential by $\varphi$. We define the correlator by the following expression

$$
\left\langle\prod_{k=1}^{s} \mathcal{O}_{J_{k}}\right\rangle_{\mathrm{g}=0}=\mathcal{N} \mathcal{B}_{g_{B}} \int_{\mathcal{M}_{0, s}} d \Omega \int[\mathcal{D} X \mathcal{D} Y]_{g_{B}} e^{-S} \prod_{k=1}^{s} \mathcal{O}_{J_{k}}\left(z_{k}(\Omega)\right),
$$

where $\mathcal{N}$ is the normalization factor and $\mathcal{B}_{g_{B}}$ are the ghost determinants, which for the sphere are just an overall factor. We parametrize the moduli space by some coordinates $\Omega$. The measure $d \Omega$ is taken to be Weyl and Diff invariant. The action $S$ is defined in (3.17). The complex number $z_{k}(\Omega)$ is the position of double pole with residue $J_{k}$ at point $\Omega$ of moduli space. Note that in this prescriptions $\mathcal{O}_{J}$ have to be $(0,0)$ operators.

The set of points with all edges integer valued in $\varphi$ metric correspond to Feynman diagrams. Note, that each diagram will appear $n(\varphi(\Omega))$ times. This is given by

$$
n(\varphi)=\frac{1}{\# \Gamma(\varphi)} \prod_{J=1}^{\infty} v_{J} !
$$

where $\# \Gamma(\varphi)$ is the symmetry factor of the diagram and $v_{J}$ is the number of vertices of valence $J$. One can understand the equality above as follows. Given a diagram we have $\prod_{J=1}^{\infty} v_{J}$ ! ways to assign labels to the vertices. However, the assignments can be grouped into equivalence classes with $\# \Gamma(\varphi)$ elements in each class. The graphs are equivalent if there is a mapping between the two sets of vertices and the two set of edges which respects the ordering of the lines at each vertex. By Strebel theorem we have a unique point in the

moduli space corresponding for each class and thus there are $n(\varphi)$ points corresponding to the diagram.

\subsection{Operators in the string theory}

The operators we will discuss take the following form

$$
\mathcal{O}_{J}=\frac{1}{4 \pi^{2}} J^{3}\left(\frac{\pi \hat{\mu}}{2}\right)^{-\frac{1}{2} J}|\Delta| e^{2 \pi i(|J|-2) X} e^{2 \pi i J Y} .
$$

The operators are not normal ordered. The normalization is chosen for later convenience. These are $(0,0)$ operators and can be used in the above described prescription. The 
two operators appearing in the definition of the action, the "puncture" and the "dilaton" operators, take the following explicit form

$$
\mathcal{O}_{0}=\int d^{2} z \sqrt{g_{D}} e^{-4 \pi i X}, \quad \mathcal{O}_{-2}=\int d^{2} z \sqrt{g_{D}} e^{-4 \pi i Y}
$$

Note that these operators are Weyl invariant as the metric $g_{D}$ is the "non-dynamical" one. Thus, there is no need to normal order the operators for this reason and we will see that there will be no need for renormalization in this prescription. In a sense the metric $g_{D}$ and the factors of $\Delta$ provide the renormalization needed for the correlators to be finite as we will see in what follows. In what follows we will refer to the factors of $|\Delta|$ in the definition of $\mathcal{O}_{J}$, and to the factors of $\sqrt{g_{D}}$ in $\mathcal{O}_{0,-2}$ as field independent terms.

\subsection{Definition of $g_{D}$ and $\Delta$ at the Strebel points}

Let us specify the functions $g_{D}$ and $\Delta$ appearing in the definitions of the worldsheet theory. We will do so only for the Strebel points. For a heuristic discussion of a generic point in moduli space see appendix B. To do so we make a short detour into properties of Strebel differentials with integer lengths.

Given a specific correlator in the string theory

$$
\left\langle\prod_{k=1}^{s} \mathcal{O}_{J_{k}}\right\rangle_{\mathrm{g}},
$$

we define the metric $g_{D}$ as follows. The set of all Strebel differentials with $s$ double poles is isomorphic to the decorated moduli space $\mathcal{M}_{\mathrm{g}, s} \times \mathbb{R}_{+}^{s}$. Thus, we parametrize the moduli space by the Strebel differentials which have the set $\left\{J_{k}\right\}$ as their residues. This is a projection of the decorated moduli space to moduli space itself. The set of the Strebel differentials, $\varphi$, satisfying the above property is isomorphic to $\mathcal{M}_{\mathrm{g}, s}$. If all the edge lengths of differential $\varphi$ are integer valued we define (as in equation $(2.8))^{8}$

$$
\begin{gathered}
\varphi_{D} d z^{2} \equiv-\frac{\varphi}{\sin ^{2} \pi l(z)} d z^{2} \\
\tilde{\varphi} d z^{2} \equiv-\frac{\varphi}{\cos ^{2} \pi l(z)} d z^{2}=-\frac{\varphi_{D}}{\cos ^{2} \pi l_{D}(z)} d z^{2}
\end{gathered}
$$

where we have used

$$
l(z) \equiv \int_{z^{\prime}}^{z} d z \sqrt{\varphi}, \quad l_{D}(z) \equiv \int_{z_{D}^{\prime}}^{z} d z \sqrt{\varphi_{D}}
$$

and $z^{\prime}, z_{D}^{\prime}$ are some given zeros of the differential $\varphi$ and $\varphi_{D}$ respectively. As was shown in [30] when all the edges of $\varphi$ are integer the differential $\varphi_{D}$ is essentially Strebel (and the

\footnotetext{
${ }^{8}$ Note that $l(z)$ has dimensions of length on the worldsheet. Thus, in equation 3.24 when $l(z)$ appears we essentially mean $l(z) / l_{0}$ with $l_{0}=1$ for convenience. If so desired the factors of $l_{0}$ can reproduced in all the equations to follow.
} 
same proof holds for $\tilde{\varphi}$ ). The above three differentials satisfy

$$
\frac{1}{\varphi}+\frac{1}{\varphi_{D}}+\frac{1}{\tilde{\varphi}}=0
$$

The critical curve of $\varphi_{D}$ is the dual graph of critical curve of $\varphi$. For the sake of graph duality we regard integer distanced points as vertices (see figure 1 for an illustration). The critical curve of $\varphi_{D}$ at a Strebel point is one of the Feynman diagrams contributing to (3.23).

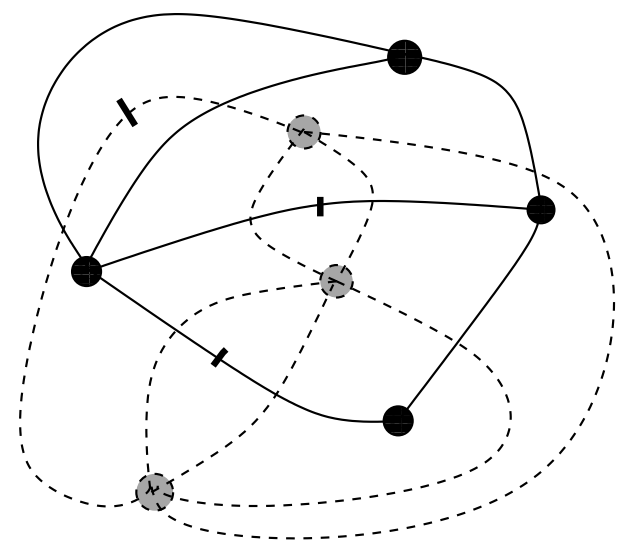

Figure 1: The solid line is an example of critical curve of $\varphi$ and the dashed line is the critical curve of corresponding $\varphi_{D}$. The notches on the edges represent integer distanced points. One can observe that the two graphs are dual to each other.

Because $l(z)$ is an integral of $\sqrt{\varphi}$ we can translate the definition (3.24) into a first order non-linear differential equation for $\varphi_{D}$,

$$
4 \pi^{2}\left(\varphi_{D}+\varphi\right)+\left(\partial \ln \frac{\varphi}{\varphi_{D}}\right)^{2}=0 .
$$

This can be recast by defining $\varphi_{D}=\varphi e^{\hat{\chi}}$

$$
(\partial \hat{\chi})^{2}+4 \pi^{2} e^{\hat{\chi}} \varphi+4 \pi^{2} \varphi=0 .
$$

Interestingly, the left hand side of the above equation looks like a "holomorphic Lagrangian" for a chiral boson $\hat{\chi}$ with a Liouville interaction, and with a meromorphic "metric" $\varphi$. We can integrate this equation for any $\varphi$ to obtain

$$
e^{\hat{\chi}}=-\frac{1}{\sin ^{2} \pi(l(z)+B)}
$$

where $B$ is some complex constant. For $B \in \mathbb{Z}$ we get $\varphi_{D}$ and for $B \in \mathbb{Z}+\frac{1}{2}$ we get $\tilde{\varphi}$, and there is a continuum of other boundary conditions. We can use 3.28 ) to define $\varphi_{D}$ for non-Strebel points, but then the solution, $\hat{\chi}$, turns out to be not single valued and 
essentially is to be defined on some covering space of the Riemann surface. Thus, (3.28) has well defined solutions only for Strebel differentials $\varphi$ with integer edges.

We define metrics corresponding to the differentials,

$$
g=|\varphi|, \quad g_{D}^{\prime}=\left|\varphi_{D}\right|, \quad \tilde{g}=|\tilde{\varphi}| .
$$

Using

$$
g=e^{2 \omega} \rightarrow \sqrt{g} R=-4 \partial \bar{\partial} \omega \quad\left(\sqrt{g_{D}} R_{D}=-2 \partial \bar{\partial} \ln \left|\varphi_{D}\right|\right),
$$

these metrics are associated with the following Ricci scalars,

$$
\begin{aligned}
-\frac{\sqrt{g} R}{2 \pi} & =\sum_{k \in P_{D} \cup Z_{D}} m_{k} \delta^{(2)}\left(z-z_{k}\right), \\
-\frac{\sqrt{\tilde{g}} \tilde{R}}{2 \pi} & =\sum_{k \in Z_{D}}\left(p_{k}-2\right) \delta^{(2)}\left(z-z_{k}\right)+\sum_{k \in P_{D}} m_{k} \delta^{(2)}\left(z-z_{k}\right)-2 \sum_{k \in E_{D}} \delta^{(2)}\left(z-z_{k}\right) \\
-\frac{\sqrt{g_{D}^{\prime}} R_{D}^{\prime}}{2 \pi} & =\sum_{k \in Z_{D} \cup P_{D}}\left(p_{k}-2\right) \delta^{(2)}\left(z-z_{k}\right),
\end{aligned}
$$

where the set $P_{D}$ is the set of double poles of $\varphi_{D}$, the set $Z_{D}$ is the set of zeros and simple poles of $\varphi_{D}$, and the set $E_{D}$ is the set of edge centers of $\varphi_{D}$. We define the numbers $m_{k}$ to be the behavior of $\varphi$ at a special point, i.e. $\varphi \sim z^{m}$. The numbers $p_{k}-2$ are the behavior of $\varphi_{D}$ at a special point, i.e. $\varphi_{D} \sim z^{p-2}$. From this we deduce that

$$
\begin{aligned}
& \frac{\sqrt{g} R-\sqrt{\tilde{g}} \tilde{R}}{4}=\partial \bar{\partial} \ln \left|\tan \pi l_{D}\right|=\pi\left[\frac{1}{2} \sum_{k \in Z_{D}} p_{k} \delta^{2}\left(z-z_{k}\right)-\sum_{k \in E_{D}} \delta^{2}\left(z-z_{k}\right)\right], \\
& \partial \bar{\partial} \ln \left|\varphi_{D}\right|=\pi \sum_{k \in Z_{D} \cup P_{D}}\left(p_{k}-2\right) \delta^{2}\left(z-z_{k}\right) .
\end{aligned}
$$

We summarize the behavior of the differentials in vicinity of special points in the following table,

\begin{tabular}{|c|c|c|c|}
\hline & $\varphi_{D}$ & $\tan ^{2} \pi l_{D}$ & $\varphi$ \\
\hline \hline$z \in Z_{D}$ & $C z^{p-2}$ & $\frac{4 \pi^{2}}{p^{2}} C z^{p}$ & $-\frac{p^{2}}{4 \pi^{2}} \frac{1}{z^{2}}$ \\
\hline$z \in P_{D}$ & $-\frac{(m+2)^{2}}{4 \pi^{2}} \frac{1}{z^{2}}$ & 1 & $B z^{m}$ \\
\hline$z \in E_{D}$ & $C$ & $\frac{1}{\pi^{2} C} \frac{1}{z^{2}}$ & $-C$ \\
\hline
\end{tabular}

where $B$ and $C$ are some complex numbers.

We are finally ready to define the quantities $g_{D}$ and $\Delta$ at the Strebel points,

$$
\begin{aligned}
& g_{D}=\frac{g_{D}^{\prime}}{g_{D}^{\prime}(\infty)}=\left|\frac{\prod_{k \in Z_{D}}\left(z-z_{k}\right)^{p_{k}-2}}{\prod_{l \in P_{D}}\left(z-z_{l}\right)^{2}}\right|, \\
& \Delta=-\frac{1}{\cos ^{2} \pi l}=\tan ^{2} \pi l_{D},
\end{aligned}
$$

where we also assumed for simplicity that $\infty \notin P_{D}$. 


\section{From strings to matrices}

In this section we will explicitly show how the worldsheet model introduced in the previous section reproduces the matrix model results. We remind that the explicit discussion is restricted to the planar topology of the worldsheet. The exact claim is that contributions from planar and connected matrix model diagrams lacking homotopically trivial self contractions to the following correlator,

$$
\left\langle\prod_{j=1}^{s} \operatorname{Tr} Q^{J_{j}}\right\rangle_{\mathrm{g}=0},
$$

are reproduced by the worldsheet correlator

$$
\left\langle\prod_{j=1}^{s} \mathcal{O}_{J_{j}}\right\rangle_{\mathrm{g}=0} \equiv \int_{\mathcal{M}_{0, s}} d \Omega \mathcal{I} .
$$

Following the procedure of section 3.2 we insert the operators $\mathcal{O}_{J_{j}}$ at the double poles of $\varphi$ (denoted in what follows by $z_{k}$ ). We denote the path integrals of the fields $X$ and $Y$ as,

$$
\begin{aligned}
& \mathcal{I}_{X} \equiv \int[\mathcal{D} X]_{g_{B}} e^{-S_{X}} \prod_{k=1}^{s} e^{2 \pi i\left(J_{k}-2\right) X\left(z_{k}\right)}, \\
& \mathcal{I}_{Y} \equiv \int[\mathcal{D} Y]_{g_{B}} e^{-S_{Y}} \prod_{k=1}^{s} e^{2 \pi i J_{k} Y\left(z_{k}\right)}
\end{aligned}
$$

We thus discuss these quantities at the Strebel points and assume that the integration over the moduli space localizes on Strebel points.

The integration over the zero mode for the $X$ field implies that in the expansion of the exponential of the interaction $\mathcal{O}_{0}$ only the product of $f$ interaction terms contributes. Here $f$ is the number of the faces of the critical curve of $\varphi_{D}$, i.e. the number of faces of the Feynman diagram corresponding to the particular Strebel point. The zero mode of the $Y$ field fixes the number of the interaction insertions $\mathcal{O}_{-2}$ to be $e$, with $e=\frac{1}{2} \sum_{k=1}^{s} J_{k}$, the number of edges of the diagram. For the above to hold it is essential that both fields $X$ and $Y$ are periodic with period 1, i.e. the zero modes take value in $[0,1]$. Using (3.32) to compute the linear terms in the $X$ and $Y$ actions we obtain the following

$$
\begin{aligned}
& \mathcal{I}_{X}=\left(\operatorname{det}^{\prime} \Delta_{g_{B}}\right)^{-1 / 2} \frac{\hat{\mu}^{f}}{f !} \int \prod_{k=1}^{f}\left[d^{2} z_{k}^{\prime} \sqrt{g_{D}\left(z_{k}^{\prime}\right)}\right] \prod_{m, n=1}^{f} \frac{\left|z_{m n}\right|^{2}\left|z_{m n}^{\prime}\right|^{2}}{\left|z_{m}-z_{n}^{\prime}\right|^{4}} \\
& \mathcal{I}_{Y}=\left(\operatorname{det}^{\prime} \Delta_{g_{B}}\right)^{-1 / 2} \frac{\hat{\tau}^{e}}{e !} \int \prod_{k=1}^{e}\left[d^{2} \tilde{z}_{k} \sqrt{g_{D}\left(\tilde{z}_{k}\right)}\right] \prod_{m, n=1}^{e} \frac{\left|\hat{z}_{m n}\right|^{2}\left|\tilde{z}_{m n}\right|^{2}}{\left|\hat{z}_{m}-\tilde{z}_{n}\right|^{4}}
\end{aligned}
$$

where $z_{k}$ are the locations of double poles of $\varphi_{D}, \hat{z}_{k}$ the locations of edge centers of $\varphi_{D}$, $z_{k}^{\prime}$ are the locations of $\mathcal{O}_{0}$, and $\tilde{z}_{k}$ are locations of $\mathcal{O}_{-2}$ interactions. We have to integrate 
the above expressions over the interaction insertions $z^{\prime}$ and $\tilde{z}$. These integrals have to be regularized, and we do so by regularizing the expressions for the Green's function needed to compute (4.4) as

$$
G\left(z, z^{\prime}\right) \sim-\ln \left(\left|z-z^{\prime}\right|^{2}+\epsilon^{2}\right)
$$

where $\epsilon$ is a small real number which will be sent to zero at the end. We also regularize the differential $\varphi_{D}$ in the vicinity of its zeros and poles as

$$
\left|\varphi_{D}\right| \sim C_{k}\left(z \bar{z}+\epsilon^{2}\right)^{\frac{1}{2}\left(p_{k}-2\right)} .
$$

Moreover note that

$$
\delta_{\epsilon, k}^{(2)}(z, \bar{z})=\frac{k-1}{\pi} \frac{\epsilon^{2(k-1)}}{\left(|z|^{2}+\epsilon^{2}\right)^{k}},
$$

is a representation of the $\delta$-function on the complex plane for any $k>1$ in the limit of small $\epsilon$.

\section{The $X$ CFT part}

First, we deal with the $X$ part of the correlator. With the above regularization we can write,

$$
\begin{aligned}
\mathcal{I}_{X} & =\left(\operatorname{det}^{\prime} \Delta_{g_{B}}\right)^{-1 / 2} \frac{\left(\hat{\mu} / \epsilon^{4}\right)^{f}}{f !} \int \prod_{l=1}^{f} d^{2} z_{l}^{\prime} \epsilon^{4 f} \prod_{m, n=1}^{f} \frac{\left(\left|z_{m n}\right|^{2}+\epsilon^{2}\right)\left(\left|z_{m n}^{\prime}\right|^{2}+\epsilon^{2}\right)}{\left(\left|z_{m}-z_{n}^{\prime}\right|^{2}+\epsilon^{2}\right)^{3}} \ldots \\
& =\left(\frac{\pi \hat{\mu}}{2}\right)^{f}\left(\operatorname{det}^{\prime} \Delta_{g_{B}}\right)^{-1 / 2} \prod_{k \in P_{D}}\left|C_{k}\right| .
\end{aligned}
$$

Here $C_{k}$ is the residue of $\varphi_{D}$ at pole $z_{k} \in P_{D}$. We used the $\delta$-function representation above with $k=3$, and take the $\epsilon \rightarrow 0$ limit. The extra power of $\left(\left|z_{m}-z_{n}^{\prime}\right|^{2}+\epsilon^{2}\right)$ comes from the $g_{D}$ factor in the definition of the puncture operator. The dots in the first line represent the regular terms coming from $g_{D}$ factor, see (3.35). Thus we get a finite result. The insertions of $\mathcal{O}_{0}$ are localized at the face centers.

\section{The Y CFT part}

Next, we deal with the $Y$ part of the correlator. With the above regularization we can write at the Strebel points,

$$
\begin{aligned}
\mathcal{I}_{Y} & =\left(\operatorname{det}^{\prime} \Delta_{g_{B}}\right)^{-1 / 2} \frac{\left(\hat{\tau} / \epsilon^{2}\right)^{e}}{e !} \int \prod_{l=1}^{e} d^{2} \tilde{z}_{l} \epsilon^{2 e} \prod_{m, n=1}^{e} \frac{\left(\left|\hat{z}_{m n}\right|^{2}+\epsilon^{2}\right)\left(\left|\tilde{z}_{m n}\right|^{2}+\epsilon^{2}\right)}{\left(\left|\hat{z}_{m}-\tilde{z}_{n}\right|^{2}+\epsilon^{2}\right)^{2}} \ldots \\
& =(\pi \hat{\tau})^{e}\left(\operatorname{det}^{\prime} \Delta_{g_{B}}\right)^{-1 / 2} \epsilon^{2 e} \prod_{k \in E_{D}}\left|C_{k}\right| .
\end{aligned}
$$

Here $C_{k}$ is the value of $\varphi_{D}$ at edge center $z_{k} \in E_{D}$. As we will see shortly there is an additional factor of $\epsilon^{-2 e}$ coming from the field independent terms which will cancell $\epsilon^{2 e}$ term above. We will get a finite result. The insertions of $\mathcal{O}_{-2}$ are localized at the edge centers. 


\section{Field independent terms}

Let us evaluate the actions $S_{L}$ and $S_{\Delta}$ and the field independent factors in the definition of the operators at the Strebel points.

We compute the Liouville action $S_{L}\left(g_{B} \rightarrow g_{D}\right)$. For concreteness we choose a simple gauge on the sphere with the metric given by

$$
\varphi_{B}=-\frac{\alpha^{2}}{z^{2}}, \quad g_{B}=\left|\varphi_{B}\right| .
$$

Because the model is Weyl invariant the result will not dependend on the detais of this metric. The Liouville factor is

$$
\omega=\frac{1}{2} \ln \left|\varphi_{D}\right|+\frac{1}{2} \ln |z|^{2}-\frac{1}{2} \ln |\alpha|^{2}
$$

and the Liouville action is

$$
S_{L}=\frac{1}{12 \pi} \int d^{2} z\left[\partial \omega \bar{\partial} \omega+\frac{1}{2} \sqrt{g_{B}} R_{B} \omega\right] .
$$

Explicitly we get

$$
\begin{aligned}
S_{L}= & -\frac{1}{48 \pi} \int d^{2} z\left[\ln \left|\varphi_{D}\right| \bar{\partial} \partial \ln \left|\varphi_{D}\right|+2 \ln |\alpha|^{2} \bar{\partial} \partial \ln |z|^{2}-\right. \\
& \left.-\ln |z|^{2} \bar{\partial} \partial \ln |z|^{2}+\ln |z|^{2} \bar{\partial} \partial \ln \left|\varphi_{D}\right|-\bar{\partial} \partial \ln |z|^{2} \ln \left|\varphi_{D}\right|\right] .
\end{aligned}
$$

The first term is given by

$$
-\frac{1}{48 \pi} \int d^{2} z \ln \left|\varphi_{D}\right| \partial \bar{\partial} \ln \left|\varphi_{D}\right|=-\frac{1}{48} \sum_{z_{k} \in P_{D} \cup Z_{D}}\left(\ln \left|C_{k}\right|+\left(p_{k}-2\right) \ln \epsilon\right)\left(p_{k}-2\right) .
$$

The number $\epsilon$ is some real, small regulator and in the end we will take it to zero. Constants $C_{k}$ are the residues of $\varphi_{D}$ at the special points, see table 3.34. Two last terms in (4.13) sum up to

$$
\begin{aligned}
& \int d^{2} z \ln |z|^{2} \bar{\partial} \partial \ln \left|\varphi_{D}\right|=2 \pi\left(\ln \left|\varphi_{D}(0)\right|-\ln \left|\varphi_{D}(\infty)\right|\right) \\
& \int d^{2} z \bar{\partial} \partial \ln |z|^{2} \ln \left|\varphi_{D}\right|=2 \pi\left(\ln \left|\varphi_{D}(0)\right|+\ln \left|\varphi_{D}(\infty)\right|\right) \\
& \rightarrow \quad-\frac{1}{48 \pi} \int d^{2} z\left[\ln |z|^{2} \bar{\partial} \partial \ln \left|\varphi_{D}\right|-\bar{\partial} \partial \ln |z|^{2} \ln \left|\varphi_{D}\right|\right]=\frac{1}{12} \ln \left|\varphi_{D}(\infty)\right| .
\end{aligned}
$$

Remember that we have set $\varphi_{D}(\infty)=1$ (see (3.35) ) and thus the above vanishes. The last term we did not discuss gives

$$
\frac{1}{48 \pi} \int d^{2} z\left(\ln |z|^{2} \bar{\partial} \partial \ln |z|^{2}-2 \ln |\alpha|^{2} \bar{\partial} \partial \ln |z|^{2}\right)=\frac{1}{6} \ln \epsilon-\frac{1}{6} \ln |\alpha|^{2} .
$$


Combining all the factors together we get

$$
-24 S_{L}=4 \ln |\alpha|^{2}-4 \ln \epsilon+\frac{1}{2} \sum_{z_{k} \in P_{D} \cup Z_{D}}\left(\ln \left|C_{k}\right|+\left(p_{k}-2\right) \ln \epsilon\right)\left(p_{k}-2\right) .
$$

From the $S_{\Delta}$ action we get the following for Strebel points

$$
\begin{aligned}
S_{\Delta} & =-\frac{2}{\pi} \int d^{2} z \partial \ln \left|\tan \pi l_{D}\right| \bar{\partial} \ln \left|\tan \pi l_{D}\right|= \\
& =\sum_{z_{k} \in Z_{D}}\left(\frac{1}{2} \ln \left|C_{k}\right|+\ln \frac{2 \pi}{p_{k}}+\frac{1}{2} p_{k} \ln \epsilon\right) p_{k}+2 \sum_{z_{k} \in E_{D}}\left(\frac{1}{2} \ln \left|C_{k}\right|+\ln \pi+\ln \epsilon\right) .
\end{aligned}
$$

Using the above we compute the finite contributions from the field independent terms, which we will denote by $\exp (\mathcal{A})$. The Liouville action, $S_{L}$ (4.17), gives the following contribution

$$
\mathcal{A}_{L}=\frac{1}{2} \sum_{z_{k} \in Z_{D} \cup P_{D}}\left(p_{k}-2\right) \ln \left|C_{k}\right|
$$

The $S_{\Delta}$ action (4.18) contributes

$$
\mathcal{A}_{\Delta}=-\frac{1}{2} \sum_{z_{k} \in Z_{D}} p_{k} \ln \left|C_{k}\right|-\sum_{z_{k} \in E_{D}} \ln \left|C_{k}\right| .
$$

For the external operators we get from (3.21) using (3.34)

$$
\mathcal{A}_{\mathcal{O}_{J}}=\sum_{z_{k} \in Z_{D}} \ln \left|C_{k}\right|
$$

and from the interactions we obtain (using the results (4.8) and (4.9))

$$
\mathcal{A}_{\mathcal{O}_{0}}=\sum_{z_{k} \in P_{D}} \ln \left|C_{k}\right|, \quad \mathcal{A}_{\mathcal{O}_{-2}}=\sum_{z_{k} \in E_{D}} \ln \left|C_{k}\right|
$$

Thus we note that the finite field independent terms cancel out at the Strebel points,

$$
\mathcal{A}=\mathcal{A}_{L}+\mathcal{A}_{\Delta}+\sum_{k \in Z_{D}} \mathcal{A}_{\mathcal{O}_{J_{k}}}+\sum_{k \in P_{D}}^{f} \mathcal{A}_{\mathcal{O}_{0}}+\sum_{k \in E_{D}}^{e} \mathcal{A}_{\mathcal{O}_{-2}}=0
$$

Next, the $\epsilon$ dependence from the field independent terms is as follows,

$$
\begin{aligned}
S_{L} & \rightarrow \frac{1}{2} \sum_{z_{k} \in Z_{D} \cup P_{D}}\left(p_{k}-2\right)^{2} \ln \epsilon-4 \ln \epsilon, \quad S_{\Delta} \rightarrow-\frac{1}{2} \sum_{z_{k} \in Z_{D}} p_{k}^{2} \ln \epsilon-2 \sum_{z_{k} \in E_{D}} \ln \epsilon \\
\mathcal{O}_{J} & \rightarrow \sum_{z_{k} \in Z_{D}} p_{k} \ln \epsilon, \quad \mathcal{O}_{0} \rightarrow-2 \sum_{z_{k} \in P_{D}} \ln \epsilon, \quad \mathcal{O}_{-2} \rightarrow 0 .
\end{aligned}
$$

These sum up to

$$
-4 \ln \epsilon-4 e \ln \epsilon+2 s \ln \epsilon=(-2 e-2 f) \ln \epsilon \text {. }
$$




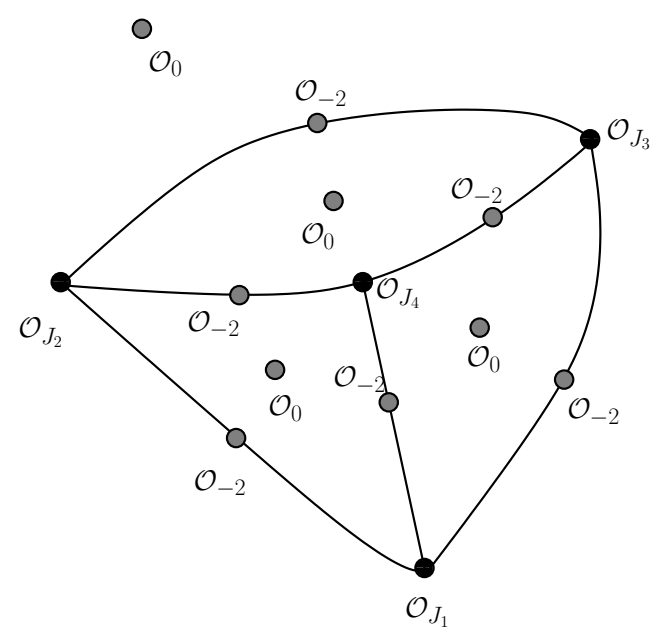

Figure 2: An example of a diagram with the interaction vertices localized at edge and face centers.

The factor $-2 f \ln \epsilon$ has been already taken into account in the $X C F T$ calculation, and the factor $-2 e \ln \epsilon$ cancels the $2 e \ln \epsilon$ contribution obtained in $Y C F T$ calculation.

To summarize, at Strebel points the calculation is finite and is precisely given by

$$
\left.\mathcal{I}\right|_{\text {Strebel }}=\mathcal{N} \mathcal{B}_{g_{B}}|\alpha|^{8}\left(\operatorname{det}^{\prime} \Delta_{g_{B}}\right)^{-1}\left(\frac{\pi \hat{\mu}}{2}\right)^{f}(\pi \hat{\tau})^{e}
$$

where $\mathcal{I}$ is defined in (4.2). Collecting all the terms and assuming that the only contributions to the moduli space integration come from the Strebel points we get

$$
\left\langle\prod_{k=1}^{s} \mathcal{O}_{J_{k}}\right\rangle_{\mathrm{g}=0}=\mathcal{N B}_{g_{B}}|\alpha|^{8}\left(\operatorname{det}^{\prime} \Delta_{g_{B}}\right)^{-1}\left(\frac{\pi \hat{\mu}}{2}\right)^{f-e}(\pi \hat{\tau})^{e} \prod_{k=1}^{s} J_{k} \prod_{J=1}^{\infty} v_{J} ! \sum_{i} \frac{1}{\# \Gamma_{i}}
$$

Identifying $\hat{\mu}=2 N / \pi, \hat{\tau}=1 / \pi t$, and choosing normalization ${ }^{9}$

$$
\mathcal{N}=\frac{\operatorname{det}^{\prime} \Delta_{g_{B}}}{\mathcal{B}_{g_{B}}|\alpha|^{8}}
$$

we get

$$
\left\langle\prod_{k=1}^{s} \mathcal{O}_{J_{k}}\right\rangle_{\mathrm{g}=0}=\left\langle\prod_{k=1}^{s} \operatorname{Tr} Q^{J_{k}}\right\rangle_{\mathrm{g}=0}
$$

\section{Summary}

Let us briefly summarize our results. We have defined the following worldsheet model. The

\footnotetext{
${ }^{9}$ Note that essentially $\mathcal{N}$ is independent of $\alpha$ due to $W e y l$ invariance.
} 
action is given by

$$
\begin{array}{lll}
c=-23 & : & S_{\tilde{X}}=2 \pi \int d^{2} z \partial \tilde{X} \bar{\partial} \tilde{X}-i \int d^{2} z \sqrt{g_{B}} R_{B} \tilde{X}-\hat{\mu} \mathcal{O}_{0}, \\
c=1 & : \quad S_{\hat{Y}}=2 \pi \int d^{2} z \partial \hat{Y} \bar{\partial} \hat{Y}-\hat{\tau} \mathcal{O}_{-2}, \\
c=48 \quad: & S_{\chi} .
\end{array}
$$

The integrated operators are defined as

$$
\mathcal{O}_{J}=\int d^{2} z \sqrt{g_{B}} e^{2 \pi i(|J|-2) \tilde{X}} e^{2 \pi i J \hat{Y}} \mathcal{O}_{J}^{(\chi)} .
$$

We do not explicitly know the action $S_{\chi}$ and the form of $\mathcal{O}_{J}^{(\chi)}$. However, assuming that this objects have certain properties we have shown that sphere correlators in the above string theory reproduce planar Gaussian matrix model correlators. The assumptions on the field $\chi$ are as follows. First, we assume that the path integral over $\chi$ localizes the moduli space integration to a discrete set of points, ${ }^{10}$ the Strebel points. Next, this path integral takes an explicit form at the Strebel points. In the language of the bulk of the paper this is responsible for the "field independent" terms in the calculation of the $X$ and $Y$ path integrals.

For the construction of this paper to be complete it will be very crucial to understand whether there actually is a $c=48 C F T$ with the above structure of correlators. As these correlators presumably depend on $\varphi_{D}$, this $C F T$ is expected to be defined through Strebel differentials or give rise to these differentials in some manner. ${ }^{11}$ We leave the investigation of these issues for future research.

\section{Acknowledgments}

I am grateful to O. Aharony, R. Gopakumar, A. Pakman, and L. Rastelli for very useful discussions and comments on the manuscript. I would like to thank the organizers of the Monsoon Workshop on String Theory and the HET Group at the Weizmann Institute for hospitality during different stages of this project. This work is supported in part by DOE grant DEFG-0292-ER40697 and by NSF grant PHY-0653351-001. Any opinions, findings, and conclusions or recommendations expressed in this material are those of the author and do not necessarily reflect the views of the National Science Foundation.

\section{A. A short primer on Strebel differentials}

A quadratic differential is the following object,

$$
q=\varphi(z) d z^{2},
$$

\footnotetext{
${ }^{10}$ In appendix B we present a heuristic suggestion for the actual localization mechanism.

${ }^{11}$ For instance, in the context of closed string field theory the Strebel differentials appear as giving rise to a minimal area metric [17].
} 
where $\varphi$ is a meromorphic function on a given Riemann surface. This differential is defined to have the following property under a holomorphic reparametrization of the worldsheet $z \rightarrow z^{\prime}(z)$,

$$
\varphi(z) d z^{2}=\varphi^{\prime}\left(z^{\prime}\right)\left(d z^{\prime}\right)^{2}
$$

Using quadratic differentials one can define a length for a line element through

$$
d l=\sqrt{\varphi} d z
$$

Note that this length is in general a complex number. It is useful to define the notions of horizontal and vertical curves of the differential. Given a curve $\gamma(t)$ on the Riemann surface we say that it is horizontal if

$$
\varphi(\gamma(t))\left(\frac{d \gamma}{d t}\right)^{2}>0
$$

and vertical if the opposite inequality holds. Note that the length of the horizontal curves computed using (A.3) is real. By convention we will discuss the horizontal curves in what follows. A horizontal curve can either be closed or end on a zero or a pole. The set of all non-closed horizontal curves of a quadratic differential is called the critical curve of the differential. We restrict to quadratic differentials critical curve of which is compact. If a quadratic differential has at most double poles (with negative coefficients) then the critical curve divides the Riemann surface into ring domains. The vertices of the critical curve of such a differential are the zeros and the simple poles of the differential. The following theorem due to K. Strebel holds,

Given a Riemann surface with $s$ marked points and $s$ positive numbers $p_{k}$ associated to those points, there is a unique quadratic differential with double poles as its only singularities such that:

- It has exactly s double poles located at the marked points

- The residues of the double poles are the numbers $p_{k}$

- The Riemann surface is a union of s disc domains defined by the marked points.

We refer to a differential which satisfies the properties above as a Strebel differential. Note that from this theorem follows that there is a unique Strebel differential for each point of $\mathcal{M}_{\mathrm{g}, s} \times \mathbb{R}_{+}^{s}$. Further, this also gives us a natural isomorphism between the space $\mathcal{M}_{\mathrm{g}, s} \times \mathbb{R}_{+}^{s}$ and the space of metric graphs with $s$ faces on genus $\mathrm{g}$ surface. ${ }^{12}$ For each point of $\mathcal{M}_{\mathrm{g}, s} \times \mathbb{R}_{+}^{s}$ we associate the critical curve of the corresponding Strebel differential

\footnotetext{
${ }^{12}$ In this context we define a metric graph as a connected graph with a positive real number associated to every edge and all the vertices at least trivalent.
} 
as the metric graph (metric on the graph defined through (A.3)), and the other direction of the isomorphism can be also (less trivially) established.

When explicitly trying to find a Strebel differential for a given Riemann surface and a given set of residues the first two conditions above can be easily satisfied. The third condition is however a very non-trivial one. Essentially, it can be rephrased as the requirement that all the distances between the zeros of the differential computed in Strebel metric (A.3) should be real. Computing these distances will give constraints on the parameters of the differential. Usually these constraints will be expressed through elliptic integrals, which are difficult to solve.

\section{B. A localization mechanism}

In this section we discuss a possible definition of the quantities $g_{D}$ and $\Delta$ for arbitrary points of the moduli space. The definition of these quantities is closely related to the mechanism responsible for the moduli space integration localizing on Strebel points.

As was discussed in the bulk of the paper for a given $s$ point correlator and a given point of the moduli space one can associate a Strebel differential $\varphi$. If not all the edges of $\varphi$ are integer valued some of the zeros of $\varphi$ are non-integer distanced. In this case (3.24) is not well defined and we have to refine it. Demanding that for Strebel points $g_{D}$ is defined as in (3.24) we can have several possible definitions of $\varphi_{D}$ at a generic point in the moduli space. We will seek a definition which has a similar structural form, i.e. $\varphi_{D}$ is a product of the differential $\varphi$ and another function defined in terms of some lengths computed using $\varphi$.

Note that at Strebel points the nature of poles and zeros of $\varphi_{D}$ as defined in (3.24) is very different. The zeros of $\varphi_{D}$ come from divergent lengths, $l(z)$, near a pole of $\varphi$. The pole combines with the divergence of the edge length and we get a zero. Thus in an extension structurally similar to (3.24) this will be usually also the case. On the other hand near a zero of $\varphi$ we get a pole at the Strebel points only because the position of the zero coincides with the point at which $l$ is an integer. This fact is very special to the Strebel points. Thus, the structure of the poles and zeros of $\varphi_{D}$ will change near the zeros of $\varphi$ for a generic refinement of (3.24).

Let us consider a simple refinement of the definition of $\varphi_{D}$ which we can only directly define in a vicinity of the zeros of $\varphi$. The special feature of this extension is that it is explicitly tractable. Although we do not know how to extend this definition over the whole moduli space it has qualitative features which are robust, i.e. appearance of extra zeros and poles near Strebel points. Of course it would be very interesting to find a definition of $\varphi_{D}$ valid for the entire worldsheet and even more interesting to come up with an argument which will favor a certain definition of $\varphi_{D}$. 
Near a Strebel point ( and a zero of $\varphi$ ) we define $\varphi_{D}$ in the following way

$$
\varphi_{D} \sim-\frac{(m+2)^{2}}{4 \pi^{2}} z^{m} \frac{\hat{z}^{m+2}+z^{m+2}}{\left(\hat{z}^{m+2}-z^{m+2}\right)^{2}} \equiv \varphi^{(m)},
$$

where

$$
\hat{z} \sim\left(\delta^{2} C\right)^{1 /(m+2)}
$$

The parameter $\delta$ is the fractional part of the distance of the zero around which we define the differential to some reference zero. Taking $\delta \rightarrow 0$ the differential $\varphi^{(m)}$ just becomes $-\frac{(m+2)^{2}}{4 \pi^{2}} \frac{1}{z^{2}}$, as is assumed for a Strebel point. The above differential is essentially Strebel differential for any value of $C$ and $\delta$. It has a pole of residue $m+2$ at $z=\infty$, a zero of valence $m$ at $z=0, m+2$ poles of residue $\sqrt{2}$ and $m+2$ simple zeros. The length between two points computed using $\varphi^{(m)}$ is given by

$$
l^{(m)}\left(z_{1}, z_{2}\right)=\left.\frac{i}{\pi}\left(\frac{1}{\sqrt{2}} \ln \left(\frac{\sqrt{2}+\sqrt{1+\left(\frac{\hat{z}}{z}\right)^{2+m}}}{\sqrt{1+\left(\frac{\hat{z}}{z}\right)^{2+m}}-\sqrt{2}}\right)-\ln \left(z^{\frac{1}{2}(m+2)}+\sqrt{\hat{z}^{2+m}+z^{2+m}}\right)\right)\right|_{z_{1}} ^{z_{2}}
$$

The critical curve of this differential for two different values of $m$ is depicted in figure 3 . The picture is that as we approach a Strebel point in the moduli space at each face of the
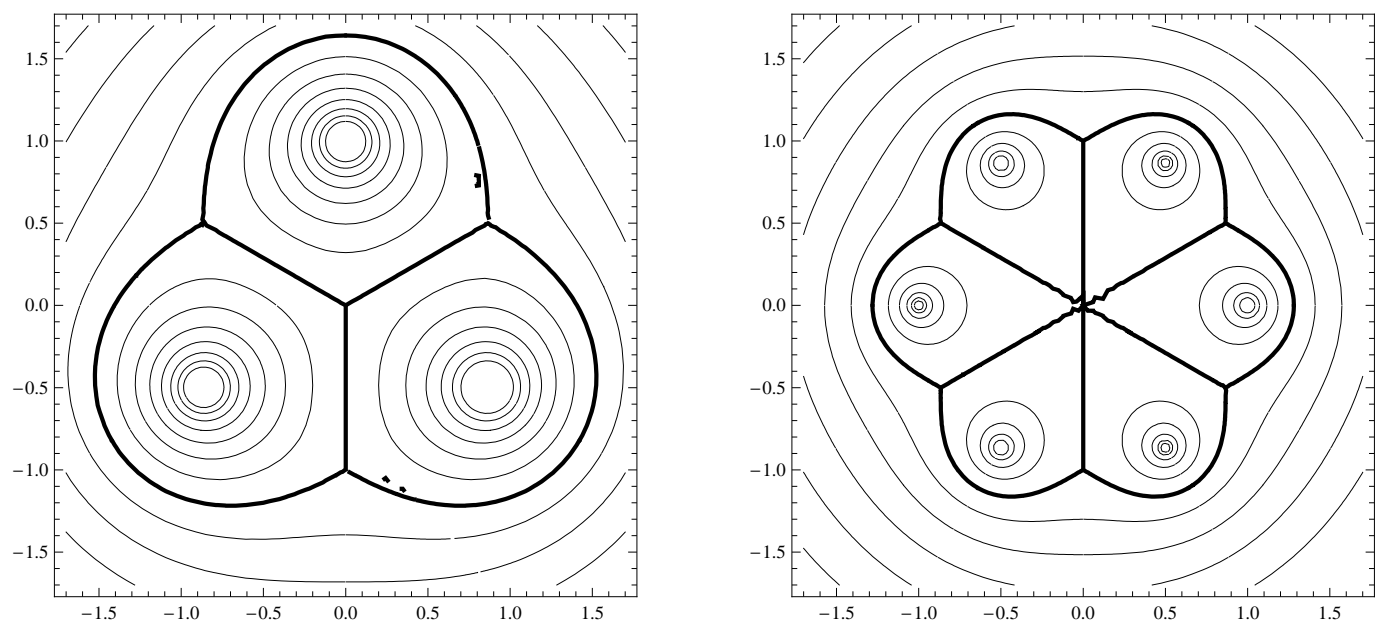

Figure 3: On the left we have $\varphi^{(m=1)}$ with $\hat{z}^{m+2}=i$, and on the right $\varphi^{(m=4)}$ with $\hat{z}^{m+2}=1$. The edges emanating from $z=0$ have length $\frac{1}{2}(\sqrt{2}-1)$, and the outer edges are all unit length (this holds for any $m$ and any $\hat{z}$ ).

diagram a sphere containing a diagram of the form depicted in figure 3 pinches off and we are left with a Strebel differential, $\varphi_{D}$, on the sphere. 
Note that the $X$ (4.8) and the $Y$ (4.9) path integrals were non vanishing because of the special structure of poles and zeros at the Strebel points. If we have additional zeros and poles, as in the above described extension to non Strebel points, these path integrals will vanish. This, provides a localization mechanism for the integration over the moduli space to the Strebel points. It would be very interesting to figure out whether the heuristic picture of this section can be made more explicit.

\section{References}

[1] R. Gopakumar and C. Vafa, On the gauge theory/geometry correspondence, Adv. Theor. Math. Phys. 3 (1999) 1415-1443, hep-th/9811131.

[2] H. Ooguri and C. Vafa, Worldsheet Derivation of a Large N Duality, Nucl. Phys. B641 (2002) 3-34, hep-th/0205297.

[3] E. Witten, Two-dimensional gravity and intersection theory on moduli space, Surveys Diff. Geom. 1 (1991) 243-310.

[4] M. Kontsevich, Intersection theory on the moduli space of curves and the matrix Airy function, Commun. Math. Phys. 147 (1992) 1-23.

[5] D. Gaiotto and L. Rastelli, A paradigm of open/closed duality: Liouville D-branes and the Kontsevich model, JHEP 07 (2005) 053, hep-th/0312196.

[6] R. Dijkgraaf, Intersection theory, integrable hierarchies and topological field theory, hep-th/9201003.

[7] P. H. Ginsparg and G. W. Moore, Lectures on 2-D gravity and 2-D string theory, hep-th/9304011.

[8] I. R. Klebanov, String theory in two-dimensions, hep-th/9108019.

[9] N. Berkovits, A New Limit of the AdS $S_{5} \times S^{5}$ Sigma Model, JHEP 08 (2007) 011, hep-th/0703282.

[10] N. Berkovits and C. Vafa, Towards a Worldsheet Derivation of the Maldacena Conjecture, JHEP 03 (2008) 031, 0711.1799.

[11] N. Berkovits, Perturbative Super-Yang-Mills from the Topological AdS $S_{5} \times S^{5}$ Sigma Model, JHEP 09 (2008) 088, 0806.1960.

[12] N. Berkovits, Simplifying and Extending the $A d S_{5} \times S^{5}$ Pure Spinor Formalism, JHEP 09 (2009) 051, 0812.5074.

[13] K. Strebel, Quadratic differentials, . Springer-Verlag, 1984.

[14] M. Mulase and M. Penkava, Ribbon graphs, quadratic differentials on riemann surfaces, and algebraic curves defined over $\bar{q}$, math-ph/9811024.

[15] D. Zvonkine, Strebel differentials on stable curves and kontsevich's proof of witten's conjecture, arXiv:math/0209071. 
[16] S. B. Giddings and E. J. Martinec, Conformal Geometry and String Field Theory, Nucl. Phys. B278 (1986) 91.

[17] B. Zwiebach, Closed string field theory: Quantum action and the B-V master equation, Nucl. Phys. B390 (1993) 33-152, hep-th/9206084.

[18] S. Mukhi, Topological matrix models, Liouville matrix model and $c=1$ string theory, hep-th/0310287.

[19] A. Okounkov and R. Pandharipande, Gromov-Witten theory, Hurwitz numbers, and matrix models. I, math/0101147.

[20] A. Okounkov, Random trees and moduli of curves, math/0309075.

[21] R. Gopakumar, From free fields to AdS. III, Phys. Rev. D72 (2005) 066008, hep-th/0504229.

[22] R. Gopakumar, From free fields to AdS, Phys. Rev. D70 (2004) 025009, hep-th/0308184.

[23] R. Gopakumar, From free fields to AdS. II, Phys. Rev. D70 (2004) 025010, hep-th/0402063.

[24] K. Furuuchi, From free fields to AdS: Thermal case, Phys. Rev. D72 (2005) 066009, hep-th/0505148.

[25] O. Aharony, Z. Komargodski, and S. S. Razamat, On the worldsheet theories of strings dual to free large $N$ gauge theories, JHEP 05 (2006) 016, hep-th/0602226.

[26] J. R. David and R. Gopakumar, From spacetime to worldsheet: Four point correlators, JHEP 01 (2007) 063, hep-th/0606078.

[27] O. Aharony, J. R. David, R. Gopakumar, Z. Komargodski, and S. S. Razamat, Comments on worldsheet theories dual to free large $N$ gauge theories, Phys. Rev. D75 (2007) 106006, hep-th/0703141.

[28] I. Yaakov, Open and closed string worldsheets from free large $N$ gauge theories with adjoint and fundamental matter, JHEP 11 (2006) 065, hep-th/0607244.

[29] J. R. David, R. Gopakumar, and A. Mukhopadhyay, Worldsheet Properties of Extremal Correlators in AdS/CFT, JHEP 10 (2008) 029, 0807.5027.

[30] S. S. Razamat, On a worldsheet dual of the Gaussian matrix model, JHEP 07 (2008) 026, 0803.2681 .

[31] A. Pakman, L. Rastelli, and S. S. Razamat, Diagrams for Symmetric Product Orbifolds, JHEP 10 (2009) 034, 0905.3448.

[32] R. C. Penner, Perturbative series and the moduli space of Riemann surfaces, J. Diff. Geom. 27 (1988) 35.

[33] S. K. Ashok, F. Cachazo, and E. Dell'Aquila, Strebel differentials with integral lengths and Argyres- Douglas singularities, hep-th/0610080.

[34] A. S. Alexandrov, A. Mironov, and A. Morozov, Instantons and merons in matrix models, Physica D235 (2007) 126-167, hep-th/0608228]. 
[35] L. Chekhov, Matrix models: A Way to quantum moduli spaces, hep-th/9305019. Matrix models and geometry of moduli spaces., hep-th/9509001.

[36] N. Itzhaki and J. McGreevy, The large $N$ harmonic oscillator as a string theory, Phys. Rev. D71 (2005) 025003, hep-th/0408180.

[37] D. Berenstein, A toy model for the AdS/CFT correspondence, JHEP 07 (2004) 018, hep-th/0403110. 